\title{
Circadian actions of melatonin at the suprachiasmatic nucleus
}

\author{
Martha U. Gillette ${ }^{\text {a*}}$, Angela J. McArthur ${ }^{1}$ b \\ a Departments of Cell and Structural Biology and Physiology, Neuroscience Program and College of Medicine, University of Illinois, \\ 506 Morrill Hall, 505 S. Goodwin Ave., University of Illinois at Urbana-Champaign, Urbana, IL 61801 USA \\ ${ }^{\mathrm{b}}$ Department of Physiology, 524 Burrill Hall, 407 S. Goodwin Ave., University of Illinois at Urbana-Champaign, Urbana, \\ IL 61801 USA
}

\begin{abstract}
The biological clock in the suprachiasmatic nucleus (SCN) of the hypothalamus plays a well-defined role in regulating melatonin production by the pineal. Emerging evidence indicates that melatonin itself can feed back upon the SCN and thereby influence circadian functions. Melatonin administration has been shown to entrain activity rhythms in rodents and humans. Melatonin binds specifically within the SCN and alters SCN physiology by both acute and clock-resetting mechanisms. The circadian clock in the SCN appears to temporally restrict its own sensitivity to melatonin, such that physiological sensitivity is greatest in the subjective dusk period
\end{abstract}

Keywords: Melatonin; Circadian rhythms; Brain slice; Pineal gland; Suprachiasmatic nucleus; Biogenic amines; Photoperiod; Neuroendocrine

\section{Introduction}

Melatonin production is regulated by a multisynaptic pathway from the biological clock in the hypothalamic suprachiasmatic nucleus (SCN) to the pineal gland (cf. [27], for review). Efferents from the SCN transmit two types of regulatory signals to the pineal. One is a permissive signal that originates within the circadian clock: it restricts melatonin synthesis to the nocturnal phase of the circadian cycle and coordinates timing of the circadian rhythm of melatonin production $[15,16,33,40]$. The second is an inhibitory signal: incidental light exposure during the night acutely interrupts the rhythm of melatonin synthesis $[12,20,34]$. Retinal afferents to the SCN provide this check on melatonin production during inappropriate light stimuli by a mechanism that antagonizes circadian regulation of the hormone's synthesis. Thus, the SCN is the primary site for both generation and integration of signals that regulate melatonin production by the pineal. Control at this central point ensures that melatonin synthesis is appro-

\footnotetext{
* Corresponding author. Fax: (217) 244-1648; email: mgillett@uiuc.edu.

${ }^{1}$ Present address: Dept. of Psychiatry, L-496, Oregon Health Sciences University, 3181 SW Sam Jackson Park Road, Portland, OR 97201, USA.
}

priately timed to coincide with both nighttime and darkness.

Accumulating evidence suggests that melatonin can itself regulate the SCN. Support for such a regulatory role comes from a variety of sources. It includes evidence that: (1) altering peripheral melatonin levels can affect behavioral rhythms; (2) specific binding of melatonin analogues at SCN sites shows circadian rhythmicity; (3) melatonin administration can acutely change SCN physiology; and (4) melatonin can directly reset the SCN circadian clock at functionally significant times. Data supporting these points will be considered in turn.

\section{Effects of altered peripheral melatonin profiles on circadian rhythms}

Studies in which melatonin was administered peripherally first suggested that melatonin might interact with the circadian timing system: the pattern and timing of melatonin injection determined the effect on behavioral rhythms. Daily intraperitoneal injection of supraphysiological concentrations of melatonin into rats in constant darkness entrained locomotory activity only if treatment directly preceded activity onset, which normally occurs at the beginning of the dark phase (night) in light-dark cycles $[2,31]$. At this subjective day-night transition 
point, melatonin caused daily activity bouts to recur with a fixed phase relation to the time of injection. When the relation between the timing of single injections was assessed against subsequent phasing of behavioral rhythms, melatonin administration at CT 10 (roughly $22 \mathrm{~h}$ after activity-onset in these rats free-running in constant darkness) induced permanent phase advance of locomotor rhythms [1]. Melatonin was largely ineffective at other times tested, although report of a responder to a predawn injection [1] suggests that sensitivity of the circadian system to melatonin might reappear shortly before dawn. More recently, peripheral melatonin administration has been found to phase shift activity cycles of mouse [5] and man $[18,20]$ at points in the late day and late night.

Further evidence that melatonin might feedback upon the SCN was provided by studies of pinealectomized rodents, in which peripheral melatonin is effectively abolished. While pinealectomy itself did not affect behavioral circadian rhythms [29], these animals entrained to a reversed lighting cycle more rapidly than those with the pineal system intact $[7,30]$. As light is the most potent entraining signal known, it is surprising that the efficacy of light increases in the absence of circulating melatonin. Also, intrinsic SCN neuronal rhythms appeared to damp in pinealectomized animals [35].

\section{Circadian changes in SCN binding of melatonin analogues}

The possibility that melatonin might cause these effects on behavioral rhythms by direct actions upon the SCN was strengthened by reports that the SCN expresses significant levels of melatonin receptors (cf. [5], for review). Although melatonin is transported widely throughout the brain and body, via the cerebrospinal fluid and blood, surprisingly few putative melatonin receptors have been identified in brain. Among the limited sites in rodent and human brain that bind the highly specific ligand $2-\left[{ }^{125} \mathrm{I}\right]$-iodomelatonin $[6]$ is the SCN $[29,39]$. Other biogenic amines (serotonin, dopamine, norepinephrine) do not compete for $2-\left[{ }^{125} \mathrm{I}\right]-$ iodomelatonin binding sites [5], nor do they elicit the biological effects of melatonin $[6,39]$.

$2-\left[{ }^{125} \mathrm{I}\right]$-Iodomelatonin binding within the SCN has been reported to change over the circadian cycle of rats reared in a 12 -h light-12-h dark cycle $[8,17,38,41]$. The pattern of $2-\left[{ }^{125} \mathrm{I}\right]$-iodomelatonin binding, measured either by quantitative autoradiography or radioreceptor binding, oscillated with a circadian rhythm; highest binding appeared during the period surrounding the day-to-night transition $[8,38,41]$ (however, see [17]). Whether sensitivity to $2-\left[{ }^{125} \mathrm{I}\right]$-iodomelatonin binding is regulated by the light-dark cycle or the circadian clock could not be determined from these studies. Nevertheless, the timing of high binding has a significant phase relation to the nocturnal peak in pineal melatonin content. Pineal melatonin production peaked in the middle of the $12-\mathrm{h}$ dark period, while the maximum binding of $2-\left[{ }^{125} \mathrm{I}\right]$ iodomelatonin by SCN preceded this by several hours. This offset in timing of the two profiles suggests that the circadian change in binding is not triggered by exposure of the SCN to melatonin.

\section{Circadian changes in acute responses of $\mathrm{SCN}$ physiology to melatonin}

A range of physiological studies have suggested that melatonin binding leads to a functional change in the SCN. Energy utilization, monitored as 2-deoxy $\left[{ }^{14} \mathrm{C}\right]$ glucose uptake by $\mathrm{SCN}$ cells, was assessed 45 min after melatonin injection at 5 points across the circadian cycle of rats in constant darkness [3]. Highly significant damping of glucose utilization was observed after stimulation in late subjective daytime (CT 6 and 10 ), whereas a modest, but significant, enhancement was observed in the subjective predawn period (CT 22). More recently, intraperitoneal melatonin injections that preceded subjective dawn (CT 23), but not at other times including subjective predusk of rats continuously in the dark, were reported to induce immunoreactivity for the immediate-early gene product, Fos, in SCN [14].

The acute response of SCN neurons in brain slices to melatonin has been assessed in rat and hamster $[21,22,36,37]$. These reports agree that direct melatonin application to $\mathrm{SCN}$ neurons can alter their firing rate. This effect is related to the circadian phase of treatment: during late day to early night, melatonin application specifically inhibited neuronal firing, whereas there was no response at the other times tested.

\section{Circadian changes in the sensitivity of SCN rhythms to phase-shifting by melatonin}

The strongest evidence for a direct action of melatonin on the SCN biological clock has been demonstrated by our studies of phase-shifting by melatonin of intrinsic SCN rhythms in vitro. We took advantage of the ability of the SCN to survive removal from the brain into a $500 \mu \mathrm{m}$ thick coronal slice of hypothalamic tissue. Although isolated in this island of tissue in vitro, the SCN continues to generate near 24-h oscillations in the ensemble of neuronal electrical activities (Fig. 1A) [10]. This rhythm is measured by brief, random samplings of extracellular activities of individual neurons longitudinally over the circadian cycle. From these activities, we derive the running average of firing rate of the population of SCN neurons. In the unperturbed slice, activity peaks at CT 7, seven hours into subjective day of the entrained lighting cycle, and at a time like that measured in vivo [13]. This peak is an easily discernable measure of 

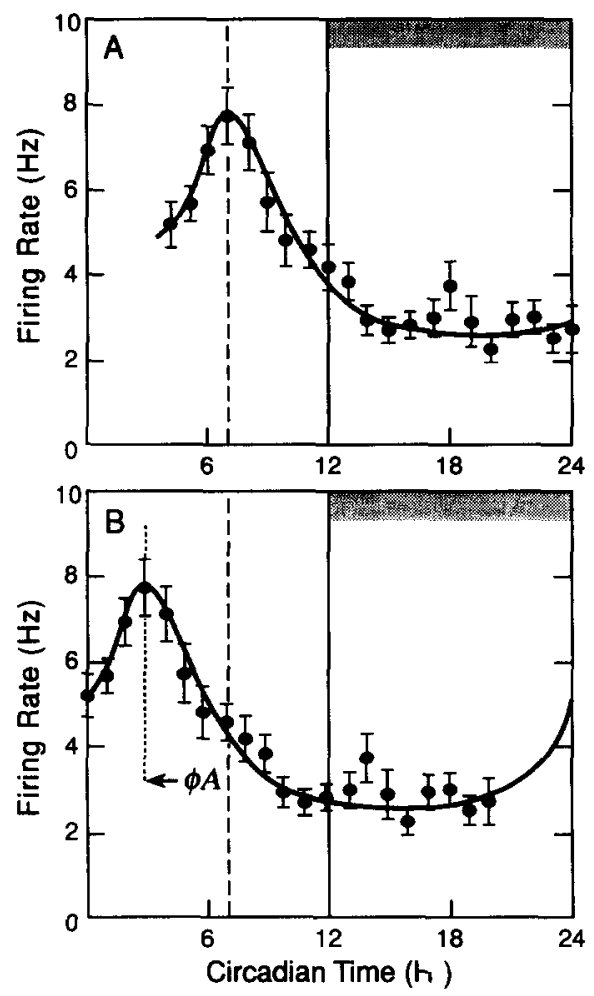

Fig. 1. Circadian rhythm in spontaneous firing rate of SCN neurons measured in the suprachiasmatic brain slice. The running 2-h mean of extracellular activity of the neuronal population is plotted against circadian time (CT) of the entrained lighting cycle of the brain-slice donor. CT 0 represents 'dawn' for the SCN clock, and thus is subjective dawn. A. The unperturbed rhythm for a brain slice prepared at CT 1.5 exhibits the typical sinusoidal pattern over the $20 \mathrm{~h}$ measured in this experiment. Activity is generally high in the subjective day (CT 0-12), and low in subjective night (CT 12-24, marked by the dark horizontal bar). Activity peaks at CT 7, marked by the dashed line; this peak is used to mark the phase of the rhythm. B. A phase-advancing stimulus, such as melatonin application at CT 10 , can shift the peak so that it appears at CT 3, four hours earlier than the peak in control slices treated with medium only (CT 7). Such a phase shift of the peak of the rhythm appears on the day after melatonin treatment and can be measured over two circadian cycles after treatment.

phase [9]. Because the tissue is under constant conditions in the brain-slice chamber, this circadian rhythm of neuronal activity is generated by the SCN's biological clock. This stable 24-h rhythm continues spontaneously for at least 3 days in a minimal salt solution supplemented only with bicarbonate and glucose [28]. In this simple preparation, responses to pharmacological agents applied directly to the SCN can be assessed unambiguously against the normally invariant basal rhythm.

Application of $1 \mathrm{nM}$ of melatonin, which is near physiological concentrations [19], directly to the SCN in this brain-slice preparation can reset the neuronal circadian rhythms [23]. When the medium in the brainslice chamber is replaced between CT 10 and CT 11 by a warmed, oxygenated melatonin solution, the time-ofpeak, and thus the phase of the rhythm, on the next day in vitro is advanced by $4 \mathrm{~h}$ (Fig. 1B). The peak appears near CT 3 on both days 2 and 3 after treatment on day 1 in vitro. Thus, the phase-advancing effect of melatonin is rapid and permanent: it is completed within the 24-h period post-treatment, after which time the rhythm continues with the new phase.

The unusual aspect of SCN sensitivity to phaseshifting by melatonin is its tight temporal restrictions within the circadian cycle [23]. Melatonin application in subjective mid-day or mid-night was without effect on SCN rhythms. However, when the SCN was exposed to melatonin during the period surrounding dusk of the entrained lighting cycle (CT 10-14), robust phase advances of $2-4 \mathrm{~h}$ occurred in subsequent neuronal rhythms (Fig. 2) [23]. Preliminary evidence suggests that surrounding subjective dawn (CT 23-1), the SCN enters a second state of sensitivity to melatonin: melatonin application within this window also induced phase advances of up to $4 \mathrm{~h}$ [24].

These effects of melatonin on the SCN circadian clock are specific. Phase advances were also induced at CT 10 by $2-\left[{ }^{125} \mathrm{I}\right]$-iodomelatonin [23]. However, application of other neurochemicals [11], including serotonin [25], neuropeptide $\mathrm{Y}$ [26] and glutamate [4], at this time did not induce the same pattern of phase shifts as melatonin. The melatonin-sensitive periods appear to bracket the transition points in the entrained lighting cycle. The transition from melatonin-insensitivity to -sensitivity occurs with an orderly progression, despite the lack of exposure to melatonin in the brain-slice chamber.

\section{Conclusions}

A remarkable feature of the emerging story of melatonin action is the consistency of the responses of the

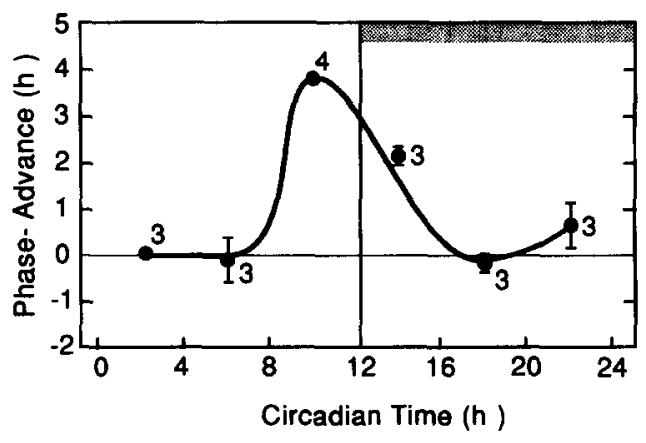

Fig. 2. The relationship between the time of melatonin application and the degree of change in the peak of neuronal activity is expressed as a phase-response curve (PRC). Evaluation of this relationship at 6 points across the 24-h cycle in vitro reveals that a 1-h exposure to $1 \times 10^{-9} \mathrm{M}$ melatonin can reset the SCN neuronal activity rhythm. Melatonin advances the next cycles of this rhythm by $4 \mathrm{~h}$ at CT 10 and $2 \mathrm{~h}$ at CT 14, but has no effect at CT 2,6, 18 and 22. ( $n \geq 3 \pm$ SEM per time point.) Subjective day and night are as in Fig. 1. (After McArthur et al., 1991 [23].) 
circadian system to this neurohormone. Despite the range of levels of analysis (organismic, systems and cellular), and the diversity of application procedures (from intraperitoneal injection to brain-slice bath) and assessment techniques (phase changes in locomotory rhythms, physiological changes in SCN metabolism in vivo, or acute and circadian changes in SCN neuronal activity in vitro), common features are apparent. Perhaps surprisingly, the SCN itself seems to undergo periodic bouts of sensitivity to melatonin near dusk and dawn. At these times, melatonin administration can adjust the phasing of the circadian clock and system.

The periods of SCN sensitivity to melatonin are distinct from SCN sensitivity to a number of other neuroactive agents [11]. They are periods during which exposure to melatonin would be minimal during the 12-h light-12-h dark cycle of laboratory animals, so sensitivity is unlikely to be a reflection of this aspect of the experimental protocol. Rather, the data suggest that melatonin production may intersect with $\mathrm{SCN}$ sensitivity only periodically. During long nights, melatonin production extends into previously crepuscular periods [32]. At these times, melatonin could close a feedback loop, regulating phasing of the circadian clock that itself times melatonin production.

\section{Acknowledgment}

Research cited from the Gillette laboratory was funded primarily by a grant (NS22155) from the National Institute for Neurological Diseases and Stroke of the National Institutes of Health.

\section{References}

[1] Armstrong, S.M., Melatonin and circadian control in mammals, Experientia, 45 (1989) 932-938.

[2] Cassone, V.M., Chesworth, M.J. and Armstrong, S.M., Entrainment of rat circadian rhythms by daily injection of melatonin depends upon the hypothalamic suprachiasmatic nuclei, Physiol. Behav., 36 (1986) 1111-1121.

[3] Cassone, V.M., Roberts, M.H. and Moore, R.Y., Effects of melatonin on 2-deoxy- $\left[1-{ }^{14} \mathrm{C}\right]$ glucose uptake within rat suprachiasmatic nucleus, Am. J. Physiol., 255 (1988) R332-R337.

[4] Ding, J.M., Chen, D., Weber, E.T., Faiman, L.E., Rea, M.A. and Gillette, M.U., Resetting the SCN biological clock: Mediation of nocturnal circadian shifts by glutamate and NO, Science, 266 (1994) 1713-1717.

[5] Dubocovich, M.L., Benloucif, S. and Masana, M.I., Melatonin receptors in the mammalian suprachiasmatic nucleus, Behav. Brain Res., (1995).

[6] Dubocovich, M.L. and Takahashi, J.S., Use of 2-[ $\left.{ }^{125} I\right]$-iodomelatonin to characterize melatonin binding sites in chicken retina Proc. Natl. Acad. Sci. USA, 184 (1987) 3916-3920.

[7] Finklestein, J.S., Baum, F.R. and Campbell, C.S., Entrainment of the female hamster to reversed photoperiod: Role of the pineal, Physiol. Behav., 21 (1978) 105-111.
[8] Gauer, F., Masson-Pevet, M., Skene, D.J., Vivien-Roels, B. and Pevet, P., Daily rhythms of melatonin binding sites in the rat pars tuberalis and suprachiasmatic nuclei; Evidence for a regulation of melatonin receptors by melatonin itself, Neuroendocrinology, 57(1) (1993) 120-126.

[9] Gillette, M.U., The suprachiasmatic nuclei: Circadian phase-shifts induced at the time of hypothalamic slice preparation are preserved in vitro, Brain Res., 379 (1986) 176-181.

[10] Gillette, M.U., SCN electrophysiology in vitro: Rhythmic activity and endogenous clock properties. In D.C. Klein, R.Y. Moore and S.M. Reppert (Eds.), SCN: The Mind's Clock, Proc. NICHD Conf., Oct. 10-12, 1989, Oxford University Press, NY, 1991, pp. $125-143$.

[11] Gillette, M.U., Medanic, M., McArthur, A.J., Liu, C., Ding, J.M., Faiman, L.E., Weber, E.T., Tcheng, T.K. and Gallman, E.A., Intrinsic neuronal rhythms in the suprachiasmatic nuclei and their adjustment. In Circadian Clocks and their Adjustment, Ciba Foundation Symp. 183, Wiley, Chichester, 1995, pp. 134-153.

[12] Illnerova, H. and Vanecek, J., Response of rat pineal serotonin $\mathrm{N}$-acetyltransferase to one minute light pulses at different times of the night, Brain Res., 167 (1979) 431-434.

[13] Inouye, S.-I.T. and Kawamura, H., Persistence of circadian rhythmicity in a mammalian hypothalamic "island" containing the suprachiasmatic nucleus, Proc. Natl. Acad. Sci., USA, 76 (1979) $5961-5966$

[14] Kilduff, T.S., Landel, H.B., Nagy, G.S., Sutin, E.L., Dement, W.C. and Heller, H.C., Melatonin influences Fos expression in the rat suprachiasmatic nucleus, Mol. Brain Res., 16 (1992) 47-56.

[15] Klein, D.C., Weller, J.L. and Moore, R.Y., Melatonin metabolism: Neural regulation of pineal serotonin $\mathrm{N}$-acetyltransferase activity, Proc. Natl. Acad. Sci., USA, 68 (1971) 3107-3110.

[16] Klein, D.C., Photoregulation of the mammalian pineal. In Photoperiodism, Melatonin and the Pineal, Ciba Foundation Symp. 117, Pittman, London, 1985, pp. 51-56.

[17] Laitinen, J.T., Castren, E., Vakkuri, O. and Saavedra, J., Diurnal rhythm of melatonin binding in the rat suprachiasmatic nucleus, Endocrinology, 124 (1989) 1585-1587.

[18] Lewy, A.J., Ahmed, S. and Sack, R.L., Phase shifting the human circadian clock using melatonin, Beh. Brain Res., 73 (1995) 121-126.

[19] Lewy, A.J., Tetsuo, M., Markey, S.P., Goodwin, F.K. and Kopin, I.J., Pinealectomy abolishes plasma melatonin in the rat, J. Clin. Endocrinol. Metab., 50 (1980) 204-205.

[20] Lewy, A.J., Wehr, T.A., Goodwin, R.K., Newsome, D.A. and Markey, S.P., Light suppresses melatonin secretion in humans, Science, 210 (1980) 1267-1269.

[21] Margraf, R.R. and Lynch, G.R., An in vitro circadian rhythm of melatonin sensitivity in the suprachiasmatic nucleus of the Djungarian hamster, Phodopus sungorus, Brain Res., 609 (1993) 45-50.

[22] Mason, R. and Brooks, A., The electrophysiological effects of melatonin and a putative melatonin antagonist ( $\mathrm{N}$-acetyltryptamine) on rat suprachiasmatic neurones in vitro, Neurosci. Lett., 95 (1988) 296-301.

[23] McArthur, A.J., Gillette, M.U. and Prosser, R.A., Melatonin directly resets the rat suprachiasmatic circadian clock in vitro, Brain Res., 565 (1991) 158-161.

[24] McArthur, A.J. and Gillette, M.U., Melatonin resets the SCN circadian clock in vitro within a narrow window of sensitivity near dawn, Soc. Neurosci. Abstr., 18 (1992) 879.

[25] Medanic, M. and Gillette, M.U., Serotonin regulates the phase of the rat suprachiasmatic circadian pacemaker in vitro only during the subjective day, J. Physiol. (London), 450 (1992) $629-642$.

[26] Medanic, M. and Gillette, M.U., Suprachiasmatic nucleus circadian pacemaker of rat shows two windows of sensitivity to neuropeptide Y In vitro, Brain Res., 620 (1993) 281-286. 
[27] Moore, R.Y. Neural control of the pineal gland, Behav. Brain Res., (1995).

[28] Prosser, R.A. and Gillette, M.U., The mammalian circadian clock in the suprachiasmatic nuclei is reset in vitro by cAMP, J. Neurosci., 9 (1989) 1073-1081.

[29] Quay, W.B., Individuation and lack of pineal effect in the rat's circadian locomotor rhythm, Physiol. Behav., 3 (1968) 109-118.

[30] Quay, W.B., Precocious entrainment and associated characteristics of activity patterns following pinealectomy and reversal of photoperiod, Physiol. Behav., 5 (1970) 1281-1290.

[31] Redman, J., Armstrong, S.M. and Ng, K.T., Free-running activity rhythms in the rat: Entrainment by melatonin, Science, 219 (1983) 1089-1091.

[32] Reppert, S.M., Weaver, D.R., Rivkees, S.A. and Stopa, E.G., Putative melatonin receptors in a human biological clock, Science, 242 (1988) 78-81.

[33] Reiter, R.J., Pineal melatonin: cell biology of its synthesis and of its physiological interactions, Endocrinol. Rev., 12(1991) 151-180.

[34] Rollag, M.D., Panke, E.S., Trakulrungsi, W.K., Trakulrungsi, C. and Reiter, R.J., Quantitation of daily melatonin synthesis in the hamster pineal gland, Endocrinology, 106 (1980) 231-236.
[35] Rusak, B. and Yu, G.-D., Regulation of melatonin-sensitivity and firing-rate rhythms of hamster suprachiasmatic nucleus neurons: Pinealectomy effects, Brain Res., 602 (1993) 200-204.

[36] Shibata, S., Cassone, V.M. and Moore, R.Y., Effects of melatonin on neuronal activity in the rat suprachiasmatic nucleus in vitro, Neurosci. Lett., 97 (1989) 140-144.

[37] Stehle, J., Vanecek, J. and Vollrath, V., Effects of melatonin on spontaneous electrical activity of neurons in rat suprachiasmatic nuclei: an in vitro iontophoretic study, J. Neural Transm., 78 (1989) 173-177.

[38] Tenn, C. and Niles, L.P., Physiological regulation of melatonin receptors in rat suprachiasmatic nuclei: Diurnal rhythmicity and effects of stress, Mol. Cell. Endocrinol., 98 (1993) 43-48.

[39] Weaver, D.T., Rivkees, S.A. and Reppert, S.M., Localization and characterization of melatonin receptors in rodent brain by in vitro autoradiography, J. Neurosci., 9 (1989) 2581-2590.

[40] Wurtman, R.J., Axelrod, J. and Phillips, D.E., Melatonin synthesis in the pineal gland: Control by light, Science, 142 (1963) 1071-1073.

[41] Zisapel, M., Nir, I. and Laudon, M., Circadian variations in melatonin-binding in discrete areas of the male rat brain, FEBS Lett., 232 (1988) 172-176. 